\title{
Corela
}

Cognition, représentation, langage

HS-29 | 2019

Questions et exclamations au prisme de plusieurs approches linguistiques

\section{L'exclamation en anglais : point de vue diachronique}

Sylvie HANCIL

(2) OpenEdition

Journals

Édition électronique

URL : http://journals.openedition.org/corela/8578

DOI : $10.4000 /$ corela.8578

ISSN : 1638-573X

Éditeur

Cercle linguistique du Centre et de l'Ouest - CerLICO

Référence électronique

Sylvie HANCIL, "L'exclamation en anglais : point de vue diachronique », Corela [En ligne], HS-29 | 2019, mis en ligne le 05 septembre 2019, consulté le 28 octobre 2019. URL : http://journals.openedition.org/ corela/8578; DOI : 10.4000/corela.8578

Ce document a été généré automatiquement le 28 octobre 2019.

\section{(c) (1) (2) (2)}

Corela - cognition, représentation, langage est mis à disposition selon les termes de la licence

Creative Commons Attribution - Pas d'Utilisation Commerciale - Partage dans les Mêmes Conditions 4.0 International. 


\title{
L'exclamation en anglais : point de vue diachronique
}

\author{
Sylvie HANCIL
}

\section{Introduction}

1 Selon l'OED (Oxford English Dictionary), l'exclamation concerne l'action de crier, d'exprimer haut et fort diverses sortes d'émotions comme la peine, la colère, la surprise, etc. :

1. The action of exclaiming or crying out; the loud articulate expression of pain, anger, surprise, etc. ; clamour, vociferation. Also, an instance of this, an outcry ; an emphatic or vehement speech or sentence, surprise, etc.; clamour, vociferation. Also, an instance of this, an outcry ; an emphatic or vehement speech or sentence.

2. The action of loudly complaining or protesting; a loud complaint or protest; a derogatory outcry ; a 'vociferous reproach' (Johnson).

(OED, troisième édition, version en ligne)

2 La réalisation la plus commune de la fonction exclamative est l'emploi du point d'exclamation (Lat. punctus exclamativus ou punctus admirativus), appelé exclamation mark en anglais britannique et exclamation point en anglais américain. Il est apparu pour la première fois au $14^{\text {ème }}$ siècle pour montrer qu'un énoncé avait besoin d'être lu avec une « certaine force exclamative » (Crystal $1996: 283$ ). Selon ce même auteur, la première forme graphique était différente dans les manuscrits les plus anciens, où le point d'exclamation était marqué par deux points en-dessous d'une ligne courte avec une barre oblique vers la droite. Néanmoins, les imprimeurs le représentaient par une ligne verticale.

3 On s'appuiera sur cette définition de l'OED et sur le texte d'anglais contemporain proposé pour s'attacher à reconstituer les principales formes d'exclamation depuis le vieil-anglais. On commencera par s'intéresser à l'apparition des interjections en vieilanglais et à la façon dont elles sont perçues à l'époque. On poursuivra ce tour d'horizon par une analyse diachronique des fonctions exclamatives des mots en wh-, en 
particulier what et how. On continuera par focaliser notre attention sur les marques d'intensité de so et that. On terminera cette étude avec l'emploi de l'impératif.

\section{Les interjections}

4 Une classe de mots dont la principale fonction est traditionnellement définie comme l'expression d'émotions est la classe des interjections, dont certaines sont illustrées dans le texte: oh (1. 28) et why (l. 66). C'est un phénomène linguistique qui relève principalement de la langue orale, mais pour le vieil-anglais, on peut seulement analyser les occurrences apparaissant dans des documents écrits, surtout dans les homélies.

5 Les interjections n'ont pas suscité le principal intérêt des grammairiens modernes ou même des spécialistes de l'anglo-saxon. Les grammaires et manuels du vieil-anglais le plus souvent ne les mentionnent pas. Par exemple, elles n'apparaissent pas dans le premier volume du Cambridge History of the English Language. Néanmoins, une source importante d'informations peut être trouvée dans la grammaire d'Ælfric, écrite autour de 1000, qui engage une discussion théorique sur les émotions en vieil-anglais.

6 En s'appuyant sur la tradition grammaticale latine, Ælfric consacre un chapitre entier aux interjections (pp.277-280, ed. Zupitza). Il définit les interjections tout à fait traditionnellement comme une classe de mots exprimant des émotions :

Interiectio est pars orationis significans mentis affectum voce incondita :

Interiectio is an dæl ledenspræce getacnjende pæs modes gewilninge mid ungesceapenre stemne.

(Ælfric's Grammar, \$277)

L'interjection est une partie du discours exprimant la disposition de l'esprit au moyen de sons informes.

(Trad. Mensah et Toupin $2005:$ 173)

7 Ælfric appelle les émotions modes gewilnung « le désir de l'esprit » ou modes styrung « le trouble de l'esprit ", qui semblent être des traductions du latin mentis affectus.

Ses termes pour L. interiectio sont betwux-aworpennyss et betwux-alegednys, littéralement « quelque chose jeté entre » ou " quelque chose mis entre »; telles sont les traductionsemprunts du terme latin interiectio. Ce sont des hapax legomena qui semblent avoir été créés par Ælfric; probablement, ils ne faisaient pas partie du vocabulaire vieil-anglais mais seulement de la terminologie d'Ælfric et ils étaient utilisés principalement pour l'enseignement de la grammaire en classe.

9 Suivant la tradition grammaticale, Ælfric mentionne aussi les principales caractéristiques des interjections, dont certaines sont encore réitérées dans les grammaires actuelles. Les voici :

(1) caractéristiques sémantiques: les interjections ont un sens (significationgetacnung) : elles expriment des émotions (modes gewilnung, etc.).

(2) caractéristiques phonologiques et morphologiques: les interjections sont phonologiquement et morphologiquement irrégulières et n'ont pas de structure ou forme fixe ; elles sont prononcées voce incondita - mid ungesceapenre stemne « avec des sons informes». De plus, Ælfric explique que les interjections peuvent être raccourcies ou rallongées selon l'état émotionnel de l'énonciateur : « ac heora sweg byð hwilon gescyrt and hwilon gelencged be ðæs modes styrung » (280/11-11-13).

(3) Caractéristiques syntaxiques: les interjections ne sont pas habituellement intégrées dans une phrase, et sont souvent avant la phrase. Ælffric dit que l'interjection est située entre les autres mots: «lið betwux wordum» (278/3), et 
c'est aussi, bien sûr, le sens de son terme dérivé betwux-alegednys (et betwuxaworpennyss).

(4) Caractéristiques interlinguistiques: Ælfric ajoute que les interjections ne peuvent pas toujours être facilement traduites $\mathrm{du}$ latin vers l'anglais (279/12-280/1). Cependant, il dit aussi que certaines interjections sont identiques en latin et en vieil-anglais (par ex. haha et hui).

Néanmoins, les caractéristiques (1) et (2) ne sont que partiellement vraies: les interjections peuvent avoir également d'autres fonctions en plus de l'expression des émotions; de surcroît, les interjections sont affectées par des changements de sons réguliers et de nombreuses interjections complexes sont créées à partir de simples interjections. En ce qui concerne la caractéristique (3), au moins l'interjection Lat. uae VA wa est parfois intégrée dans la phrase. Ælfric ajoute que les interjections sont comme des mots : pes deel interiectio hoefo wordes fremminge (279/12) : il les traite comme une des huit classes de mots ou parties du discours.

11 Les émotions qu' Ælfric mentionne, et qui peuvent être exprimées par des interjections sont : la joie (modes bliss), la tristesse et la détresse (modes sarnyss), l'émerveillement et l'étonnement (wundrung), la peur (oga), la colère (cebylignyss, yrre, yrsung), la repentance (exprimée verbalement par behreowsian), le mépris (forsewennyss), le dédain (bysmerung, les lamentations (wanung), les menaces peow-wreec-peowracan), les insultes (wyrigung)

Ælfric note aussi que certaines interjections sont polysémiques et peuvent exprimer des émotions tout à fait différentes, par exemple L. euge: joie et dédain (bliss and bysmerung). Lat. $o$ a même 5 fonctions (280/3-5) : (1) le mot exprime la colère (abilignyss ); (2) la peine (sarnyss); (3) l'étonnement (wundrung); (4) mais il exprime aussi le vocatif ("adverbium vocandi »): o magister - eala ðu larew; (5) et il remplace la lettre $<0$.

Cela montre aussi deux autres phénomènes: (a) toutes les fonctions des interjections n'expriment pas des émotions ; (b) un mot peut appartenir à plusieurs classes de mots $o$ peut être une interjection et un adverbe (d'après Ælfric); une lettre (stcef), assurément, a encore un statut différent.

14 D'un point de vue étymologique ea (comme dans eala), wa, la, hui, et haha sont des interjections anciennes, datant du germanique et même de l'indo-européen :
(a) $e a>$ Gmc *au $>$ IE cf. L au
(b) haha $>$ Gmc *haha > IE cf. L. haha
(c) hui > Gmc *hui (cf. All. hui) > IE cf. L. hui
(d) la : vieil-all. le et L. il-le
(e) wa > Gmc * wai cf. All. Weh(e) > IE cf. L. vae

15 La plupart des interjections complexes semblent cependant être des formations vieilanglaises. Bien que les grammaires soulignent souvent que les interjections sont des sons naturels ou en tout cas d'origine onomatopéique et qu'elles sont phonologiquement et morphologiquement irrégulières, les exemples montrent que les interjections avaient souvent des formes conventionnelles.

En s'appuyant sur la grammaire d'Ælfric et les Soliloques, quatre fonctions peuvent être distinguées pour les interjections, dont voici les plus fréquentes :

(1) les marqueurs d'émotions : (a) émotions positives : haha/hehe pour le rire; hui pour la surprise et l'admiration; (b) émotions négatives, exprimant la tristesse, le regret, le chagrin, etc. (apparemment pour une grande majorité) : eala, hilahi, la, wa, wais me/wamme, wala, walawa, wellawell

(2) les marqueurs qui attirent l'attention : hwæt 
(3) les formes pour saluer : hui(g)

(4) les formes pour répondre : afcestla, gea, na/ne/nese.

En tout, en vieil-anglais, environ 35-40 interjections peuvent être identifiées ; voici les 10 principales interjections :

(1) afcestla : 'certainly, assuredly'

(2) eala : 'alas, oh, lo' (très fréquent)

(3) gea : 'yes' (commun en vieil-anglais)

(4) haha/hehe : 'ha! ha!' (indiquant le rire)

(5) hilahi : 'alas, oh'

(6) hui.huig : pour l'admiration ou la surprise ou les deux

(7) hwcet : marqueur attirant l'attention du co-énonciateur (très fréquent)

(8) la: 'oh, ah'; fréquemment utilisé en combinaisons comme afcestla, eala, hilahi, wala, wellawell, walawa, wella.

(9) na, ne, nese : 'no' (commun)

(10) wa : 'alas'

Après la période de 1400, les changements deviennent apparents. Les marqueurs vieilanglais hwcet, la et wa survivent en tant que what, lo et wo. Les contextes favorisant leur utilisation restent les mêmes en moyen-anglais. Par exemple, Taavitsainen note que ces exemples apparaissent principalement dans des citations de discours direct imitant le langage parlé (Taavitsainen 1997: 575). D’après Taavitsainen (1998: 600), les interjections les plus fréquentes sont : al(l)as; lo/loo;o/oo; $a$; what; pardee. Mais ce ne sont pas seulement les types de mots qui changent en moyen-anglais; les genres se multiplient aussi. En particulier, le rôle des textes dits imaginatifs devient plus proéminent en moyen-anglais. On peut le voir dans le Helsinki Corpus avec l'émergence de genres comme les histoires d'amour en vers, les lettres et le théâtre dramatique, ainsi que la croissance de la fiction en prose.

\section{Les marqueurs en wh-}

Intéressons-nous à présent aux marqueurs en wh- utilisés comme exclamatifs, illustrés dans le texte 1.19 (Haa-ha!) et 1.170 (how well you're looking).

\subsection{What}

\subsubsection{Etymologie de what}

Dans l'Oxford Concise Dictionary of English Etymology (OCDEE), what est défini comme un pronom interrogatif attesté pendant la période vieil-anglaise, et comme un adjectif interrogatif attesté pour la première fois au $12^{\text {ème }}$ siècle. Selon Barber (1999:95), en Proto-Indo-Européen (PIE), il y avait une série de plosives labialisées, à savoir $\mathrm{G}^{\mathrm{w}} \mathrm{h}, \mathrm{g}^{\mathrm{w}}$, et $\mathrm{k}^{\mathrm{w}}$. La consonne PIE $\mathrm{k}^{\mathrm{w}}$ est devenue en Proto-Germanique (PG) $/ \mathrm{hw} /$ : correspondant au latin quod, on trouve le vieux-saxon hwat et le vieil anglais hwcet. Selon An AngloSaxon Dictionary (AASD), hwcet est une forme neutre de $h w \bar{a}$; il est utilisé comme adverbe ou interjection :

(8) Hwæt iudas het ða settan dæt lic

Ah! Then Judas bade them put down the body

H. R. 13, 26)

De plus, la première occurrence de what en tant que pronom relatif date du 12ème siècle, tandis que le pronom indéfini fut identifié au $13^{\text {ème }}$ siècle. What est décrit par 
Klein (1971: 825) dans A Comprehensive Etymological Dictionary of the English Language (ACEDEL) comme étant un mot utilisé comme pronom, adjectif, nom et adverbe. Le pronom hwcet fut associé au sens de what, qui, d'après le Dictionary of Word Origins (DWO) a son origine en Indo-Européen *qwod, qui a aussi produit en Latin quod 'what'. Puis, en moyen-anglais, d'autre changements morphologiques se passèrent et petit à petit, les ligatures ${ }^{1}$ disparurent et l'initial $h$ dans $h w$ fut prononcé $/ \chi w /$ comme le wh dans de nombreux dialectes écossais (Wright $1925: 1-9$ ).

\subsubsection{What : son fonctionnement en tant qu'exclamatif}

L'éventail des fonctions de what confirme que les propositions exclamatives en wh-sont au centre des préoccupations discursives de l'utilisateur au cours des siècles. Outre la reconnaissance répandue du fonctionnement de what en tant que pronom interrogatif, il est possible d'identifier les autres fonctions incluant le renforcement, l'emphase ou l'intensification du sens émotionnel. Cette multifonctionnalité est illustrée dans le Tableau 1 :

23 A. Pronom interrogatif dans des questions directes (c. 888-présent)

B. Exclamatif- en tant qu'interjection (c. 1000- présent)

C. Exclamatif - marqueur pour attirer l'attention du co-énonciateur (c. 1000-c. 1386)

D. Exclamatif - dans une exclamation (c. 1200- c. 1886)

E. Exclamatif - marqueur d'intensité (c. 1420- c. 1865)

F. Exclamatif - marqueur pour appeler quelqu'un (c. 1386- c. 1878)

G. Exclamatif - en tant qu'adverbe (c. 900- c. 1556)

H. Exclamatif - en tant qu'adjectif (c. 1315 - c. 1888)

I. Exclamatif - dans des propositions dépendantes (c. 1300 - c. 1926)

J. Exclamatif - en tant que pronom (c. 1382 - c. 1460)

K. Pronom relatif (c. 1200 - présent)

L. Adjectif (c. 1200 - présent)

M. Conjonction (c. 1175- c. 1690)

N. Emploi d'indéfini (c. 1175- c. 1870)

Tableau 1 : Les fonctions du pronom what au cours des siècles dans l'OED et le MED (d'après Mrochen $2009: 63)$

\subsubsection{What en vieil-anglais et en moyen-anglais}

D'après le $M E D^{2}$, what a commencé de fonctionner dans une exclamation associée à une question. Par exemple :

(9) Hwot la! Hwy ett eower larðeaw mid byssen manfullen mannen \& synfullen?

'Alas! What, why is your teacher eating with the evil and sinful men?'

(a1150(OE) Vsp.D.Hom.(Vsp D.14) 59/15)

(10) What ! nis heo no3ht icome?

'What! Has she not arrived?'

(a1300(c1250) Floris (Vit D.3) 361)

Qui plus est, ce même dictionnaire inclut des exemples référant à la surprise, à la détresse ou à l'indignation. Soit :

(11) Hwet, weneð pas ruperes ...pet crist heom wulle milcien ?

'What ! The robbers think... that Christ will have mercy on them ?'

(a1225( ?OE) Lamb.Hom.(Lamb 487)) 
(12) Hwat, heo seyde, hule, artu wod?

What!' she said, 'Owl, are you mad?

(c1275( ?a1216) Owl \& N.(Clg A.9)1298)

On peut remarquer que what avec une fonction exclamative était suivi d'un point d'interrogation, ce qui veut dire qu'une variante elliptique a commencé d'être plus usitée. Soit :

(13) Quat? Wenis bu i be a fole?

'What ? Dost thou think I am a fool ?'

(a1400 Cursor (Trin-C R.3.8) 10456)

Au même moment, what continuait de fonctionner parfois comme une exclamation, parfois comme une question. Par exemple :

(14) What! shal she fyghte with an hardy knyght?

'What ! Shall she fight with a fearless knight?'

(c1430(c1386) Chaucer LGW (Benson-Robinson) 1800)

(15) What ?...ys youre harte up now?

'what ! ... Is your heart up now ?' ((a1470) Malory Wks.(Win-C) 750/7)

Brinton (1996: 187-8) propose que hwoet en vieil-anglais établit un lien d'intimité ou une distance entre énonciateur et co-énonciateur, est un marqueur pour attirer l'attention, offre une évaluation du point de vue narratif, ou bien rend le matériel plus saillant. De surcroît, il établit une bonne relation entre énonciateur et co-énonciateur avec déférence et respect. Au cours du 10ème et du $11^{\text {ème }}$ siècles, l'emploi adverbial de what fut adopté, et ainsi davantage de possibilités étaient disponibles. Par exemple :

(16) Juliana! hwoet pu gloem hafast.

'Juliana, ah! what thou hast radiant beauty' (900) (Jul 167)

Juliana, ah! How radiant your beauty is !

(17) Ei, ei, what this nicht is long!

'Hey, how long this night is!' (c1225 Mirie it is (Rwl G.22) 5)

Le pronom interrogatif what avec un sens adverbial est presque équivalent à how et voudrait dire To what an extent! In what a way! (WD, 1913). Selon WD (1913), what était parfois relié à des adjectifs avec un sens adverbial et fonctionnait presque comme how, comme dans What partial judges are our love and hate! La même fonction est discutée dans l'OED et peut être illustrée de cette façon :

(18) A god huet we hedde guod wyn yesteneuen and guode metes.

'Oh God, what good wine and good food we had yesterday evening.'

((1340) Ayenb.(Arun 57) 51/8)

(19) A ! lorde, what the wedir is colde!

'Oh lord, how cold the water is!' (a1450 Yk.Pl.(Add 35290) 114/72)

Les formes en what peuvent aussi fonctionner à travers d'autres moyens rhétoriques :

elles introduisent de manière emphatique une affirmation dans la narration, par exemple :

(20) Hwcet pa se Élmihtige Scyppend forgeaf pan seofen halgen.

'What the Almighty Creator excused the seven saints'

(a1150(OE) Vsp.D.Hom.(Vsp D.14) 25/29)

Brinton (1996: 187-8) énumère plusieurs fonctions de hwcet en vieil-anglais (qui est similaire à you know en anglais moderne) :

(i) functions to call the attention of the hearer to the following discourse,

(ii) to suggest that the information to follow is common, shared, or familiar, and 
(iii) to bring that information to consciousness, renew interest in it, make it salient or 'newly relevant', or focus attention on its importance to the following discourse, that is, to foreground it. bons exemples de couleur exclamative aussi bien que de propositions exclamatives marquées. What fonctionnait encore comme une exclamation associée à une question mais il a commencé à être suivi par des formes lexicales (noms, marques d'intensité, etc.) et un point d'exclamation. Soit :

(21) What fy! Schold I a fundeling for his fairenesse tak?

'What ! should I take a foundling for his fairness?' (a1375 Wpal.(KC 13) 48)

(22) What deuyl! Why hap pe prest swych hy?'

'What the Devil, why is the priest in such a hurry?'

(a1400(c1303) Mannyng HS (Hrl 1701) 4284) (25), par exemple :

(23) Fy what ! a lord breke his byheste or bond?

'What, a lord breaks his promise or commitment?' (a1450(1412) Hoccl. RP (Hrl 4866)

2243)

(24) Kyng ? What the dewyll, other then I ?' (a1500(a1460) Towneley Pl.(Hnt HM 1)

150/307)

(25) Ys hit thus!... what in the deuyllys date!

'Is is so! ... what in the name of the Devil!' (c1500( ?a1475) Ass.Gods (Trin-C R.3.19)

425) d'exclamation ou un point d'interrogation. La position initiale de what n'a pas changé de manière significative mais il était précédé d'autres interjections comme $A$ ! ou $O$ ! pour rendre toute la structure plus emphatique. Par exemple :

(26) O swete leuedy, wat pe was wo, po ihesus deyde on rode!

'Oh sweet lady, what pain you endured it was that Jesus died on the cross!'

(Shoreham 119 (1315))

(27) What a fawte it was, The seruaunte, alas, His master to forsake!

'What a fault it was, the servant, alas, forsakes his master !' (Digby Mist 1157 (1485))

Cet exemple s'apparente à l'exclamation en «what $a$ » du texte d'anglais contemporain : « what a convenient location » (1. 19-20).

De plus, c'est à la fin de la période moyen-anglaise qu'apparut pour la première fois what utilisé comme un adjectif pour exprimer la surprise. La construction inversée comme dans la question directe, par exemple What a place is this! était employée avec ou sans article indéfini. Cependant, selon l'OED, la construction inversée pouvait être remplacée par la construction à la forme déclarative.

Puis, à la fin du moyen-anglais, what a acquis une autre fonction et a commencé à être associé avec une exclamation soulignant emphatiquement la réponse d'un énonciateur à une situation. En même temps, c'était une exclamation appelant l'attention du coénonciateur ou exigeant sa réponse ou une réaction puisque what était accompagné d'autres expressions, comme why, here now, now listen to this. Par exemple:

(28) Ther gan oure hoost for to iape and pleye, And seyde, 'Sires, what! Don is in the myre!' 'There did our host begin to jape and play, And he said: "Sirs, what! Dun is in the mire !'

(c1390) Chaucer CT.Mcp.(Manly-Rickert) H5) 
(29) Say, felowes, what ! fynde yhe any feest, Me falles for tu haue parte, parde!

'Say fellows what - have you found any feast ? It fits I should have my part, indeed !'

(a1450 Yk.Pl.(Add 35290) 119/44) utilisée à partir du $17^{\text {ème }}$ siècle. Quelques formes mineures d'emplois rhétoriques de what fonctionnant comme une interjection apparurent au début du $15^{\text {ème }}$ siècle, par exemple :

(31) What ! pay brayen \& bleden, bi bonkkez pay degen.

'What, they, roaring and bleeding, fell dying on the banks.'

(c1400( ?c1390) Gawain (Nero A.10) 1163)

(32) Quat! Hit clattered in pe cliff... What! hit wharred \& whette as water at a mulle; What!

hit rusched \& ronge, rawpe to here.

'What! it rang in the cliff ... What! it whirred and whetted like water on a mill-

wheel; what ! it rushed

and rang, terrible to hear.'

(c1400( ?c1390) Gawain (Nero A.10) 2201,2203-4)

D'après le MED, what fut utilisé comme une exclamation dans des expressions adversatives comme but , but; but what of that, but no matter, but lo. Ces expressions étaient en position initiale, médiane ou finale. Par exemple :

(33) Sorwefully he siketh, But what ! he may nat doon al as hym lilketh.

'and sorrowfully he sighed ; But what ! He could not do as he pleased.'

(c1395) Chaucer CT.WB.(Manly-Rickert) D.914)

(34) Criseyde is now agon; But what, she shal come hastiliche ayeyn!

'Criseyde is gone now ; but what, she shall soon come again.'

(a1425(c1385) Chaucer TC (Benson-Robinson) 4.1318)

(35) Hit greuyth me, but what ! hit more, parde, Me hurt.

'It angers me, but what, it indeed hurt me more.' (c1450 ? C.d'Orl. Poems (Hrl 682)

14/409)

Il est important de noter que dans les éditions modernes, ces expressions sont spécifiées dans la structure syntaxique entre virgules ou points virgules tandis que la phrase complexe est suivie d'un point d'exclamation.

\subsubsection{What en anglais moderne}

Au début du $16^{\text {ème }}$ siècle, selon l'OED, les formes en what fonctionnaient comme des exclamations de surprise ou d'étonnement fréquemment répétées en position initiale, par exemple :

(36) What, what, latine in the mouth of a plaine fellow? (1589) (R.Harvey Pl.Perc.

(1590) 5)

Aux $17^{\mathrm{ème}}$ et $18^{\text {ème }}$ siècles, what a continué de fonctionner comme une exclamation de surprise précédée d'autres mots en wh- qui étaient employés avec une fonction d'interjection, par exemple :

(37) 'O, Mr.Jones, I have lost my Lady for ever.' - 'How ! what ! for Heaven`s Sake

tell me.

(1749) (Fielding Tom Jones xv.vii) 

expression d'excitation, par exemple :

(38) What Tibet, what Annot, what Margerie. Ye sleepe, but we doe not. (1589) Udall Royster D. i.iii (Arb) 22)

What to say ? Tibet, Annot Margerie ! You sleep, but we do not.

(39) What ? courage sirs my felowes al. (1581) (A.Hall Illiad ii.29)

Come on! Let's have some courage, my dear fellows !

Puis, pour héler ou appeler ou attirer l'attention d'une personne, what fonctionnait comme une exclamation/interjection encore utilisée de manière proéminente en position initiale, comme dans :

(40) Chamberlain, call in the music, What ! we'll make a night of it.

(1607) (Dekker \& Webster Northir. Hoe v.i.)

(41) What Ariell ; my industrious seruant Ariell. What would my potent master?

Here I am.

(1610) (Shakes.Temp.iv.i.33)

$\mathrm{Du}$ point de vue du marquage, les formes en what étaient suivies d'un point d'exclamation, d'un point d'interrogation ou d'un point-virgule. De plus, what en tant qu'interjection pouvait suivre une proposition exclamative, soit :

(42) Here they are both! What Sirs, desputin. (1633) (B.Jonson Tale Tub i.iii.)

La fonction exclamative de what était parfois attachée à des adjectifs avec un sens adverbial, presque équivalents à how. Soit :

(43) What shocking times we live in! (1798) (G.Hay Ushaw Mag.(1913) Dec.288)

(44) What rebellious they were. Mod.Sc. What bonny! (1556) (Chon. Grey Friars

(Camden) 60)

En termes de questions en wh-, what était utilisé comme adjectif : how remarkable, how great, avec une construction inversée comme dans une question directe. Dans cette construction, le mot en what était suivi de l'article indéfini a/an (WD 1913). Par exemple :

(45) What a strain'd unnatural Similitude must this seem to a Modern Reader ?

(1705) (Addison Italy 307)

(46) What a house! What people! What manners !

(1776)(Earl Carlisle in Jesse Selwyn \& Contemp.(1844)III.160)

La force exclamative de what utilisée dans des propositions dépendantes varie tout comme la force interrogative dans l'emploi interrogatif correspondant mais, selon l'OED, what s'est déplacé vers la position médiane avec des verbes de pensée ou de perception. Par exemple :

(47) When I consydere ever what servants of God they were and so dyed.

(1554) (Engl.Hist.Rev.(1913) July 528)

(48) Me thought what paine it was to drowne.

(1594) (Shakes. Rich. III, i.iii. 21)

(49) Do'st thou forget From what a torment I did free thee ?

(1610) (I Temp. i.iii. 251)

51 A la fin du $16^{\text {ème }}$ et du $17^{\text {ème }}$ siècles, what fonctionnait encore comme une exclamation dans la construction suivante. Soit :

(50) $O$ what a Cyte, and what a se royall Hath had first name of pore men and rurall.

(1509) (Barclay Shyp of Folys (1874)II.105)

(51) What a piece of worke is a man! (1602) (I Ham.ii.ii.315)

Corela, HS-29 | 2019 

nom+ structure inversée (auxiliaire + verbe + objet). De plus, pour ce qui est de la très grand force exclamative, les formes en what peuvent être précédées d'autres interjections, par exemple $O$ (voir (51)), et toute l'affirmation peut être suivie d'un point d'exclamation, un point d'interrogation ou bien un point. Cependant, selon l'OED, la construction inversée peut être utilisée dans un style archaïque (comme dans une question directe): What a place is this!, What place is this ?, ou bien à la fin de l'anglais moderne peut être transformée en What a place this is! devil, dickens, etc., what in the name of..., what in the world, what on earth, etc.). A la fin du 17 ème siècle et au début du $18^{\text {ème }}$ siècle, il y avait encore des structures intensives comme What the deuce!, Thinking what in the universe, ou I wonder what in blue thunder qui étaient utilisées pour rendre les formes en wh-plus emphatiques. Par exemple :

(52) What the deuyll ! Can ye agre no better? (1520) (Skelton Magnyf. 795)

(53) What a duce, must a man be always writing!

(1754) (Richardson in J.Duncombe Lett. (1773) III.13)

Il est à noter qu'un seul exemple de what existe dans des questions rhétoriques, impliquant une assertion contraire, délimitée par un point d'exclamation.

(54) With what becoming Thanks can I reply ! (1697) (Dryden Æneis xi. 770)

A la fin de l'anglais moderne, what était encore employé dans des propositions dépendantes, après des verbes de pensée ou de perception. Par exemple :

(55) You cannot imagine what a parcel of cheating brutes the work people here are.

(1708) (Caldwell Papers (Maitland Club) I.26)

(56) We may see after what a different manner Strada proceeds. (1713) (Addison Guardian No.119 31)

A la fin du $19^{\text {gème }}$ siècle, what fut utilisé pour héler ou attirer l'attention d'une personne, par exemple What ho (Webster's 1828 Dictionary). Pour exprimer l'aspect surprenant d'une chose ou d'une personne, what pouvait fonctionner comme une exclamation avec un sens adjectival, par exemple:

(57) 'What a consternation of soul was mine that dreary afternoon! Yet in what darkness, what dense ignorance, was the mental battle fought!'

(1847) (C.Brontë J.Eyre ii)

(58) 'Oh, what a dawn of day ! How the March sun feels like May!'

(1855) (Browning A Lovers` Quarrel i)

(59) 'What rubbish you talk.'

(1888) (Rider Haggard Col. Quaritch xli)

What était aussi fréquemment usité dans une forme elliptique, spécialement pour le reste de la question, par exemple une forme courte pour 'What did you say?' ou 'What is it ?'. Soit :

(60) 'Oh ! oh !- I'm so frightened !' 'What at, dear ?- what at ?' said the mother.

(1834) (Dickens Sk. Boz, Steam Excurs.)

(61) 'What's your name ?' 'Cold punch', murmured Mr.Pickwick, as he sunk to sleep

again.

'What?' demanded Captain Boldwig. No reply.

(1837) (I Pickw.xix)

58

Même s'il fonctionne comme un explétif interrogatif (parfois avec eh), il est souvent placé à la fin de la phrase, marquée par un point d'exclamation, en particulier dans des emplois de conversation triviale ou affectée, comme dans (62): 
(62) But then, she`s so beastly chic, dontcherknow - eh, what! (1891) (J.S.Winter Lumley xv)

(66) Good-bye, Miss Thornton, awfully jolly evening---what? (1906) (Mansfield Girl \& Gods xvi)

(67) Can't say I've read it. It's a bit too literary for me. What ? But they say it's jolly clever. You had it at school, I dare say. What?

(1914) (A.M.N. Lyons Simple Simon i.i. 16)

marquer l'emphase ou attirer l'attention (par exemple, let me tell you), what est utilisé dans une forme elliptique signifiant 'what it is', 'what is the truth of the matter', 'what is the thing to do' dans I'll tell you what, par exemple:

(68) 'You know what, Chris ?' 'What ?' 'You know it stinks to heaven as well as I do.'

(1982) (H.Engel Murder on Location xviii. 164) or 'how astonishing', par exemple :

(69) What a fool! (2004) (Dictionary.com).

\subsection{How}

\subsubsection{Etymologie}

pronom interrogatif how a subi des changements graphémiques et phonémiques et a commencé graduellement à se comporter comme une exclamation/interjection (Wright 1925 : 248-249, Pyles 1971 : 138). Selon le Dictionary of Word Origins, how appartient à une grande famille de mots utilisés pour les questions et qui ont commencé leur vie comme $q w-$. Le descendant phonétique de ceci en germanique préhistorique était ${ }^{*} k h w-$, qui en anglais moderne est représenté par wh-. Le marqueur how vient lui-même de l'adverbe germanique occidental *khwō formé à partir de la base qui a aussi produit les marqueurs anglais what et who; tout comme who, il a perdu le son /w/, mais puisque who ne l'a perdu que bien plus tard, la graphie en wh en est témoin.

Selon le Concise Dictionary of English Etymology, how a encouru les mêmes changements morphologiques concernant la lettre initale $w$ que le wh. La première forme signifiant “ in what way, by what means' attestée en vieil-anglais dans hü devint en vieux frison hū, hō 


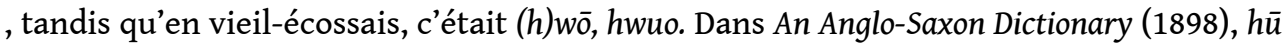
est défini comme un pronom trouvé dans les exclamations, par exemple :

(70) $h \bar{u} l a$ ! Ne gewarp une tō ānum peninge

How now ! was not our agreement for a penny?

(Th. 50,$7 ;$ Gen. 805)

Dans le A Comprehensive Etymological Dictionary of the English Language (1966 : 747, 1971 : 355), il est noté que how vient du vieil-anglais $h \bar{u}$, et est apparenté au vieux germanique hwio, au germanique wie, au gothique haiwa et au moyen-anglais hou, hu.

\subsubsection{How : le fonctionnement du marqueur en tant qu'exclamation}

CDW (2000 : 289) affirme que how fut formé en vieil-anglais à partir du germanique, à partir de la base de who, what. Dans le AASD (1898: 564-565), how est défini apparenté en tant qu'adverbe qui fonctionne dans les questions directes, cependant la seconde fonction mentionnée concerne les exclamations, par exemple dans :

(71) eala gæsta God, hu pu gleawlice mid noman ryhte nemned wære Emmanuhel

Oh! God of spirits, how rightly was thou named by the name of Emmanuel !

Le marqueur a commencé sa vie en étant utilisé dans des exclamations directes et autour de 900 pour la première fois dans le sens 'In what way! To what an extent or degree!' et dans des questions dépendantes et des exclamations principalement qualifiantes et des adverbes et adjectifs signifiant 'to what extent ; in what degree'.

Avant d'explorer tout l'éventail des fonctions des formes en how, le spectre de ses fonctions est illustré ci-dessous :

71 A. Exclamation exprimant l'étonnement : c. 1200-1450

B. Exclamation attirant l'attention : c. 1377-1825

C. Exclamation exprimant un cri de marins : c. 1450-1867

D. Exclamation exprimant un cri de douleur/peine : c. 1575-1750

E. Pronom interrogatif : c. 1000-présent

F. Exclamations directes : c. 900-1808

G. Questions et exclamations dépendantes : c. 1000-présent

$\mathrm{H}$. Introduisant une relative : c. 1400-1879

I. Interjection : c. 1877-1962

Diachroniquement parlant, on peut noter qu'il y a eu un éventail large des différentes fonctions depuis le début. Molencki (1990: 28-29) remarque que même la classification en vieil-anglais cause de nombreux problèmes. Ces structures peuvent être ambiguës quant à la distinction entre l'exclamation et l'interrogation. Il note que le choix des modes et/ou de l'ordre des mots n'aide pas non plus, par conséquent la classification peut seulement se faire sur la base de la sémantique, qui du fait de l'absence des énonciateurs natifs est souvent difficile à déterminer.

D'après l'OED, how fut employé comme un élément fonctionnant comme une interjection/exclamation autour de 1000 et il fut utilisé comme un équivalent à What ? ou What ! Par exemple :

(72) $\mathrm{Hu}$ ! haue ge wrong.

'How, you are wrong.'

(a1325(c1250) Gen. \& Ex. (Corp-C 444) 3077)

Pour les exclamations directes, how fonctionnait avec le sens de In what a way!, to what an extent or degree!, par exemple : 
(73) Hu god is ece God!

How good is eternal God ! 3

(Ags Ps (Th) Ixxii (1000))

(74) Low, huw hali writ spekeð

Lo, how the holy scripture speaks

(c1230 (a1200) Ancr. (Corp-C 402) 31/18)

(75) Hu lutel wile we beð her, hu longe ells ware !

How little time we will be here, how long anywhere else !

(c1250 PMor. (Eg 613(1)) 327)

(76) 0 how wonderful and how worpi ... a ping is ... god!

Oh, how wonderful and how worthy ... a thing is ... God!

(c1475(c1445)Pecock Donet (Bod 916) 85/35)

D'après les exemples ci-dessus, le mot how apparut dans la structure syntaxique how + adjectif/adverbe, par exemple how long, how good and how pleasant, how well, how wonderful and how worthy, qui a survécu jusqu'à maintenant.

How était employé en tant qu'exclamation exprimant l'étonnement, l'indignation, la détresse etc. ; par exemple :

(77) Hu dele ! Leuestu \& luuest te pe reufulliche deide \& reuliche on rode ?

How ! Do you believe in and do you love the one who pitifully and miserably died on the cross?

(c1225( ?c1200)St. Marg. (1) (Bod 34) 8/27)

(78) 'Alas, sir, how ? what may that be ?' 'She ys ded !'

'Alas, sir how ? What may that be ?'She is dead.'

(c1450(1369) Chaucer BD (Benson-Robinson) 1307)

Le MED répertorie certains exemples de how fonctionnant comme une exclamation introduisant une question négative, par exemple :

(79) La, hu, næ pæ pe blinde mon pe swa iboren wæs?

Lo, how, was it not the blind man who was born blind?

(c1175( ?OE) Bod. Hom. (Bod 343) 60/2)

(80) La, hu, ne beað pa pet here specað galileisce?

Lo, how, are not these who speak here Galileans?

(a1225(OE) lamb. Hom. Pentec. (Lamb487) 89)

D'après Hoey (1997 : 148), l'intensification et la focalisation donnent de la proéminence à l'élément placé en position initiale. Occasionnellement, la, lo sont placés en tête de phrase avant hu, how; par conséquent, l'effet sémantique est simplement d'intensifier puisque lo est défini comme une exclamation qui, dans son emploi ancien, signifie 'look, see' et est ajouté pour montrer que les gens sont sur le point de mentionner quelque chose d'intéressant et surprenant (CIDE 1995: 832). La même fonction d'une exclamation, mais cette fois avec le sens d'attirer l'attention du co-énonciateur, fut identifiée pour how à la fin du $15^{\text {ème }}$ siècle ; par exemple :

(81) How, gyb, good morne! wheder goys thou? Thou goys ouer the corne. Gyb, I say, how

Ow, Gyb, good morning! Whither are thou going? You are walking across the corn!, I say, how !

(a1500(a1460) Towneley Pl.I (Hnt HM 1) 102/81-82)

80 A la fin du 14ème siècle, how a commencé à être utilisé comme une exclamation et elliptiquement dans how now? dans le sens de How is it now? ; par exemple : 
(82) What, how now ... hap Clarioun my cosyn aslawe pe man?

What, how now ... has my cousin Clarion killed the man?

(c1380 Firumb. (1) (Ashm 33) 3779)

(83) What how nowe, manace ye me?

What how now, do you threaten me?

81 Aux 14ème et 15ème siècles, how fut employé comme élément dans des cris variés, comme pour la chasse, en empruntant au français, par exemple :

(84) And they trowe wele to fynde hym, ye shul saye, 'Here, how, here, douce, how, here ...'

And they truly hope to find him, so you should cry, 'Here, how, here, dear, how, here ...'

(c1425 Twiti Venery(l) (Vsp B. 12) 153)

Et dans des cris de marins, quand ils tiraient la corde ou bougeaient en rythme, par exemple :

(85) Y howe ! trussa ! Hale in the brayles !

How! Truss! Pull the ropes!

(c1500 Men may leue (Tri-C R.3.19) 33)

La même fonction est discutée dans l'OED où how indique un cri de marins soulevant l'ancre etc. ; comme par exemple :

(86) The master commandeth. To hys shypmen. With 'howe! Hissa !' then they cry.

The master gives orders ... to his crewmen ... then they cry 'how ! Hiss!'

(c1500 Men may leue (Trin-C R.3.19) 13,19)

Ou bien il fait référence à un appel pour attirer l'attention ou réveiller un dormeur ; ou une salutation, par exemple :

(87) Thanne wol I clepe, how Alison! how John! Be myrie for the flood wol passe anon.

Then I will call, 'Ho, Alison! Ho, John ! Be cheery, for the flood will pass anon.'

(c1390 Chaucer CT. Mil. (Manly-Rickert) A. 3577)

(88) How, how ! A minstrel! Know 3e ony out?

'How, how ! A minstrel! Do ye know any?'

(c1475 Mankind (Folg V.a 354) 444)

(89) Than ... Iudas ... kist his mowth \& seid, 'How hey ;'

Then ... Judas ... kissed his mouth and said, 'how hey'

(a1500 NPass. (Cmb Ff.5.48) 5446 fn)

La forme interrogative de how fut aussi utilisée au début du $15^{\text {ème }}$ siècle dans le refrain d'une chanson :

(90) banne..somme..songen atte nale And hulpen erie his half acre with 'how ! trolliloll !'.

'then some sang over the ale and helped plough his half acre crying 'how! trolli-

lolli !"

(c1400(c1378) PPl.B (LdMisc 581) 6.118)

86 A la fin du $15^{\text {ème }}$ siècle, how représentait le hululement d'un hibou :

(91) Whatt byrdys ast thou? Non but the howlat, that kreye, 'How, how !'

'What birds hast thou? None but the owl that sings 'how-how'

(a1475 Holy berith beris ( Hrl 5396) p. 94)

En anglais moderne, how était toujours utilisé comme exclamation attirant l'attention du co-énonciateur :

(92) Mak roume, sirs, hoaw! that I may rin ! (1535) (Lyndesay Satyre 602)

(93) Howe, howe, who is heare ? I Robin of Doncastere and Magaret my feare.

(1579) (Epit. in Miller Hist. Doncaster) 

modernes de What? ou What !. Par exemple :

(94) Whow? I go about to disgrace thee ? (1589) (R. Harvey Pl. Perc. (1860) 11)

(95) Elb.My wife Sir? whom I detest before heauen, and your honour. Esc. How ? thy wife ? Elb. I sir.

(1603) (Shakes. Meas. For M. ii.i. 71)

Une autre forme elliptique how now? pour how is it now? était aussi employée en interjection, par exemple :

(96) How now? moodie? (1610) (Shakes. Temp. i.ii. 244)

90 A la fin du $17^{\text {ème }}$ siècle et au début du $18^{\text {ème }}$ siècle, how était encore utilisé comme interjection et c'était la forme elliptique de How is it? ou How say you ?. D'après l'OED, how prit la position initiale de la phrase et était un marqueur tout seul suivi d'un point d'exclamation ou un point d'interrogation :

(97) How! signior have you not authority? (1722) (De Foe Col. Jack (1840) 306)

(98) 'How', cried I, 'relinquish the cause of truth ?' (1766) (Goldsm. Vic. W. xi)

91 Dans les exclamations directes, how fonctionnait en position initiale dans le sens de In what a way!:

(99) O how sweet it smelleth. (1583) (Hollyband Campo di Fior 307)

(100) My God, how endless is thy love! (1707) (Watts Hymn)

(101) How pale his cheek, his eye how bright! How you do like to tease one ! (1808)

(Scott Marm. iii.vi)

Tout au long du début de l'anglais moderne, how était encore utilisé comme cri des marins, quand ils tiraient une corde ou bougeaient en rythme, comme cri de douleur ou de détresse. Par exemple :

(102) Mony marynair Besy at thair werk ...with mony heis and how. (1513) (Douglas Æneis iii.ii. 120)

(103) Wyth, Hey, and wyth howe, Sit we down arow. (a1529) (Skelton E. Rummyng 289)

De plus, d'après le MED, how était une interjection employée par les bergers et qui commença à apparaître au début du $16^{\text {ème }}$ siècle :

(104) Howe! Haroye! how! how! dryve the sheepe to the low! thou may not heare but if I blowe.

'How! Help! How! How! take the sheep to the valley! Thou may not hear me unless I blow'

(1607( ?a1425) Chester Pl.(Hrl 2124) 134/45)

Les formes how, howe étaient utilisées pour crier how! avec peine ou chagrin dans certains dialectes :

(105) What need ye hech and how, ladies? What need ye how for me?

(c1750) (Mary Hamilton xiii. in Child Ballads (1889) III. 392)

\section{L'intensité avec so et that}

L'intensité dans la forme exclamative peut aussi se manifester par l'emploi des marqueurs so et that, illustré 1.69 et 1.131 dans le texte. Selon l'OED, l'adverbe d'intensité so a vu ses premières occurrences dès la fin du $9^{\text {ème }}$ siècle, alors que le marqueur that avec une valeur intensive date de la moitié du $15^{\text {ème }}$ siècle :

"So: In affirmative clauses, tending to become a mere intensive without comparative force, and sometimes emphasized in speaking and writing. 
c888 Ælfred tr. Boethius De Consol. Philos. xxxv. \$3 Nu ðu pæt swa openlice ongiten hæfst, ne ðearfe ic nu...ymb ðæt swincan.

OE Beowulf 347 Gif he us geunnan wile, pæt we hine swa godne gretan moton. a1225 Leg. Kath. 171 pe wrecches pet ha seh swa wraðe werkes wurchen.

1340 R. Rolle Pricke of Conscience 4073 pe empire, pat was swa myghty, Es now destruyed a grete party.

141226 Pol. Poems xi. 50 God dede pe make, Put soule of resoun in flesche so frele.

?1504 S. Hawes Example of Vertu sig. gg.ii, Amonge the floures so swete of ayre.

1741 S. Richardson Pamela III. xxix. 168 My Face...was hid in my Bosom, and I looked so silly!

1882 E. A. Floyer Unexplored Baluchistan 302 The absence of ruined buildings, which so invariably form the major part of a Persian town."

(OED, troisième edition, version en ligne)

"that : To that degree ; so much, so. Qualifying an adj, adv. or ppl. Now dialog. And Sc. ; also colloq. With a negative : not (all) that, not very.

?1450 Life ST. Cuthbert (1891) 1. 6279 His sekenes pat encrust, He gert beere him. Aboute pe contre on a bere.

1616 in J. Russell Higs of Bemersyde (1881) vii. 160 If I had been that unhappy as to have such a foolish thing.

a1670 H. Hacket Scrinia Reserata (1693) II. 67 This was carried with that little noise that the Bishop was not awakened.

1803 Boswell Change Edinb. 5 Gowd's no that scanty.

1853 Dickens Edwin Drood ii. 4 Mr. Jasper was that breathed.

[...]

1977 Spare Rib may 16/1 It's not that easy in a place like Sheffield.

1980 S. Brett Dead Side of Mike xvii. 173 Charles found it difficult to get that excited."

(OED, troisième édition, version en ligne)

Les exemples sont le résultat d'un changement décrit par Bolinger (1972: 61ff.) comme étant un changement sémantique de l'identifieur vers l'intensifieur. Les emplois des démonstratifs n'identifient plus un degré par l'intermédiaire d'un geste (emploi exophorique) ou en le reliant à un antécédent (emploi anaphorique), mais fonctionnent comme "boosters", indiquant un haut degré de qualité, ce qui donne une qualité exclamative aux énoncés les incluant. Contrairement aux démonstratifs dont ils sont dérivés, ils n'expriment plus la similarité, mais une valeur extrême sur une certaine échelle.

Une reconstruction possible du développement historique de ces phrases est susceptible de les relier à un genre particulier de construction comparative dans laquelle le degré d'une propriété est identifié à travers la capacité ou non d'un référent à manifester une certaine situation :

(106) Charles is so tall that he can reach the ceiling in the house.

98 Les exclamatives pourraient alors être des généralisations sémantiques et des réductions syntaxiques de comparatives appelées souvent « consécutives ».

\section{L'impératif}

Intéressons-nous pour finir à l'impératif, illustré 1.15 (Turn that over-light off! Turn that off!) dans le texte. L'impératif est aussi un marqueur d'exclamation et est utilisé depuis le vieil-anglais. L'impératif est un des trois modes: l'indicatif, le subjonctif et l'impératif. En vieil-anglais, l'emploi de l'impératif est réservé à l'expression des ordres 
et est restreint à la deuxième personne du singulier et du pluriel. Morphologiquement, il est marqué par $-\varnothing$ et - $a p$ respectivement.

$\mathrm{Le}^{4}$ verbe est habituellement en début de phrase, bien qu'un adverbe puisse le précéder. Dans les phrases affirmatives, le sujet est absent dans les constructions réflexives. Le subjonctif hortatif employé pour donner des suggestions n'est pas utilisé à la première personne du singulier. Cependant, il est employé aux autres personnes. Habituellement, le verbe est en première position mais l'ordre sujet-verbe peut apparaître dans les constructions à la troisième personne dans les propositions principales.

(107) Ne yldan we na from dæge to dæge

Not let-us delay we not from day to day

(HomU 37 (Nap 46))

Let us not delay from day to day.

(108) God us helpe.

May God help us

(Algeo $2010: 102$ )

101 Parce que l'impératif et le subjonctif contrastent morphologiquement, on doit assumer qu'il y avait une différence sémantique, au moins au début du vieil-anglais, entre des énoncés plus ou moins directifs, plus ou moins hortatifs. A la période Alfrédienne, cette différence a perdu du terrain dans de nombreux registres; néanmoins, le subjonctif a contribué d'être préféré dans les règles monastiques et juridiques; les charmes, les ordonnances médicales et les instructions généralisées sont normalement au subjonctif.

En moyen-anglais, les emplois du subjonctif pour l'expression de l'hortatif sont similaires.

D’autre part, le verbe à l'impératif précède aussi le sujet :

(109) Naske 3e of cunseil

Not-ask you of counsel

(Ancrene Riwle 58.569)

Dans le cas des impératifs à la forme négative, le sujet précède not et le verbe précède le sujet :

(110) Ne hide bou no3t fram me byn comaundement.

Ne hide you not from me your commandment.

(The Earliest Prose Psalter 146.2169)

L'ordre des mots en moyen-anglais des impératifs montre aussi que le verbe à l'impératif occupe le premier argument.

Au début de l'anglais moderne $e^{5}$ l'utilisation du subjonctif pour exprimer l'optatif est largement restreint aux formules comme God forgive him, Lord help our understandings, heaven grant, God save, long live, etc. Le subjonctif optatif est souvent remplacé par une périphrase, may et le subjonctif hortatif par let :

(111) 'A god rewarde you,' quoth this roge ; 'and in heauen may you find it.'

(Helsinki Corpus, Harman 39)

Des deux périphrases, celle remplaçant le subjonctif hortatif se développe moins rapidement : Dans Marlowe, à la fin du $16^{\mathrm{ème}}$ siècle, la périphrase hortative est bien plus fréquente que le subjonctif, en particulier à la première personne du pluriel, tandis que la périphrase optative est moins commune que le subjonctif. 
Pour la même période de l'anglais moderne, les impératifs montrent le même ordre des mots qu'en moyen-anglais. Mais, cette fois, les impératifs avec le soutien de do sont attestés. Dans les impératifs avec un sujet et le soutien de do, l'auxiliaire précède le sujet (ex. 112). Dans les impératifs avec un sujet mais sans soutien de do, le verbe précède le sujet (ex 113) :

(112) Do you and your fellows attend them in.

(361 M : 5 1-106)

(113) doubte thou not all things tightly orderd be.

(356 mt 90-25)

En anglais actuel, les impératifs à la forme négative requièrent l'emploi de do ; do et la négation doivent précéder le sujet (ex. 114):

(114) Don't worry.

\section{Conclusion}

111 Pour conclure, l'étude diachronique des formes exclamatives en wh-, ainsi que celle des interjections et des impératifs, montrent que les valeurs sémantiques et pragmatiques associées à ces marqueurs étaient présentes depuis le vieil-anglais pour la plupart, ce qui corrobore l'existence d'une continuité dans le développement de la langue.

A la lumière de la fonction exclamative des formes en wh-, il se trouve que le contexte situationnel, à savoir par exemple les structures syntaxiques précédentes, les ajouts d'intensité, les marqueurs honorifiques etc., a influencé la couleur émotionnelle et la force illocutoire de l'énoncé. D'autres contextes ont aussi été influencés au cours de l'histoire par différentes variables comme la position sociale, la relation de pouvoir et de solidarité entre énonciateur et co-énonciateur. Tous ces éléments sont autant de paramètres qui pourraient enrichir une recherche future sur l'exclamation.

\section{BIBLIOGRAPHIE}

Algeo, J. (2010). The origins and development of the English language. Sixth edition. Wadsworth.

Ayto, J. (1990). Dictionary of Word Origins. (DWO) London : Bloomsbury.

Barber, C. (1999). Early Modern English. Edinburgh University Press : Edinburgh.

Bolinger, D. (ed). (1972). Intonation. Selected Readings. Great-Britain : Penguin Books.

Bosworth, J. \& T. Northcote Toller (1898) [1972]. An Anglo-Saxon Dictionary.

(AASD) Oxford : Oxford University Press.

Brinton, L. J. (1996). Pragmatic Markers in English : Grammaticalization and Discourse Functions. Berlin : Mouton de Gruyter. 
Cambridge International Dictionary of English (CIDE) (1995). Cambridge : Cambridge University Press.

Crystal, D. (1996). The Cambridge Encyclopedia of The English Language. Cambridge : Cambridge University Press.

Hoad, T.F. (ed.). (1986). Concise Dictionary of English Etymology. (OCDEE) Oxford : Oxford University Press.

Hoey, M. (1991). Patterns of Lexis in Text. Oxford : Oxford University Press.

Hoye, L. (1997). Adverbs and Modality in English. London and New York : Longman.

Klein, E. (1971). A Comprehensive Etymological Dictionary of the English Language.

(CEDEL) (ACEDEL) Amsterdam : Elsevier.

Mensah, M. \& Toupin F. (2005). La Grammaire d'Ælfric. Traduction et commentaires linguistiques. Paris : Publications de l'Association des Médiévistes Anglicistes de l'Enseignement Supérieur.

Molencki, R. (1990). HW-Clauses in Old English. In Linguistica Silesiana, 12, pp. 25-48.

Oxford English Dictionary (OED) (1991). Third edition. Disponible en ligne www.oed.com.

Onions, C. T. (1966) [1964]. The Oxford Dictionary of English Etymology. (ODEE). Oxford :

The Clarendon Press.

Pyles, T. (1971). The Origins and Development of The English Language. New York-Chicago-San

Francisco-Atlanta : Harcourte Brace Jovanovich, Inc.

Room, A. (2000). Cassell`s Dictionary of Word Histories. (CDW) Great-Britain : Cassell.

Taavitsainen, I. (1997). ‘ « By Saint Anne » : Pious oaths or swearing in Middle English ? An assessment of genres', in Raymond Hickey and Stanislaw Puppel (eds.), Language History and Linguistics Modelling. A festschrift for Jacek Fisiak in his 60th birthday, Berlin : Mouton de Gruyter, 815-826.

Taavitsainen, I. (1998). ‘Emphatic Language and Romantic Prose : Changing Functions of Interjections in a Sociocultural Perspective'. European Journal of English Studies 2/2. Swets and Zeitlinger. 195- 214.

Webster's Revised Unabridged Dictionary (1913). (WD).

Wright, J. \& Wright, E. M. (1925). Old English Grammar. Oxford : Oxford University Press.

\section{NOTES}

1. Une ligature est la fusion de deux graphèmes d'une écriture pour n'en former qu'un seul nouveau, considéré comme un caractère per se.

2.

https://quod.lib.umich.edu/cgi/m/mec/med-idx?

type $=$ byte $\&$ byte $=241015192 \&$ egdisplay $=$ compact \&egs $=241024052$

3. Cet exemple se rapproche de l'exclamation qui apparaît à la 1.170 du texte de Tennessee Williams (« how well you are looking !»)

4. Ce passage est inspiré de Traugott E.C. : The Cambridge History of the English Language 1 (§ 4.3.1.3, p. 184-185).

5. L'exemple suivant est de Rissanen M., The Cambridge History of the English Language 3 (§ 4.3.3.1. p. 228-229). 


\section{RÉSUMÉS}

L'analyse de l'exclamation d'un point de vue diachronique fait l'objet de notre étude dans cet article. Le point de départ de l'analyse est la définition de l'oxford English Dictionary et on se propose de citer les principales formes exclamatives depuis le vieil anglais et de comprendre comment elles sont analysées à cette époque. On poursuit l'étude historique par une analyse diachronique des fonctions exclamatives des mots en wh-, en particulier what et how puis on s'intéresse aux marqueurs d'intensité so et that pour finir par l'emploi de l'impératif.

The analysis of exclamation from a diachronic point of view is the object of this study. The starting point is the definition of the Oxford English Dictionary and the main exclamative markers are studied in Old English and interpreted. The words in wh-, especially what and how, are the focus of our attention, followed by the analysis of markers of intensity so and that, along with the use of the imperative.

\section{INDEX}

Keywords : exclamation, wh-, intensity, imperative, diachrony

Mots-clés : exclamation, wh-, intensité, impératif, diachronie

\section{AUTEUR}

SYLVIE HANCIL

Université de Rouen

Equipe ERIAC

hancilfr@yahoo.fr 